\title{
Limited value of the standard 8-hour water deprivation test in the diagnostic work-up of patients with suspected diabetes insipidus
}

-Penelope Trimpou, Daniel S Olsson and Oskar Ragnarsson

-Department of Endocrinology, Institute of Medicine at Sahlgrenska Academy, University of Gothenburg

\section{Background}

Diabetes insipidus (DI) is characterized by polyuria and consequently thirst and polydipsia.

Excessive water intake can also cause polyuria, i.e. primary polydipsia. It is of utmost importance to differentiate between the two disorders since the treatment is different.

\section{Purpose}

To study the clinical value of the standard 8-hour as well as an extended water deprivation test in patients with polyuria and polydipsia

\section{Patients and Methods}

This was a retrospective, single centre study were results from 117 water deprivation tests performed between 2004 and 2014 were reviewed. The same protocol was used during the entire study period. Consumption of any liquids during the test was strictly prohibited.

Weight, urine osmolality and specific gravity were measured on every occasion the patient urinated throughout the test.

Following criteria were used for termination of the test:

a) $>3 \%$ weight reduction

b) Urine specific gravity $>1.020$ or, urine osmolality $>800 \mathrm{mOsm} / \mathrm{L}$

c) Intolerable adverse symptoms such as excessive thirst

\section{Aetiology of polydipsia-polyuria syndrome in 117 patients who performed water deprivation test between 2004 and} 2014

\begin{tabular}{lc} 
Primary polydipsia & 96 \\
\hline Diabetes insipidus after pituitary surgery & 4 \\
\hline Nephrogenic diabetes insipidus & 4 \\
\hline Idiopathic neurogenic diabetes insipidus & 4 \\
\hline Other* & 9 \\
\hline * Langerhans cell histiocitosis, Sheehans syndrome, hypophysitis, Wegeners &
\end{tabular}

* Langerhans cell histiocitosis, Sheehans syndrome, hypophysitis, Wegeners granulomatosis, familial diabetes insipidus.

\section{Results}

Of 117 patients (70 women, 47 men), 21 (18\%) were diagnosed with DI and 96 (82\%) with primary polydipsia.

The median (interquartile range; range) time to termination of the test was 14 hours $(10-16 ; 3-36)$ in patients with DI and 18 hours $(14-24 ; 7-48)$ in patients with primary polydipsia $(P=0.009)$.

In only $4(20 \%)$ patients with DI and $5(5 \%)$ patients with primary polydipsia the diagnostic criteria were met in less than 8 hours.

Of those diagnosed with primary polydipsia, 26 (27\%) did not reach either urine specific gravity $>1.020$ or urine osmolality $>800 \mathrm{mOsm} / \mathrm{L}$.

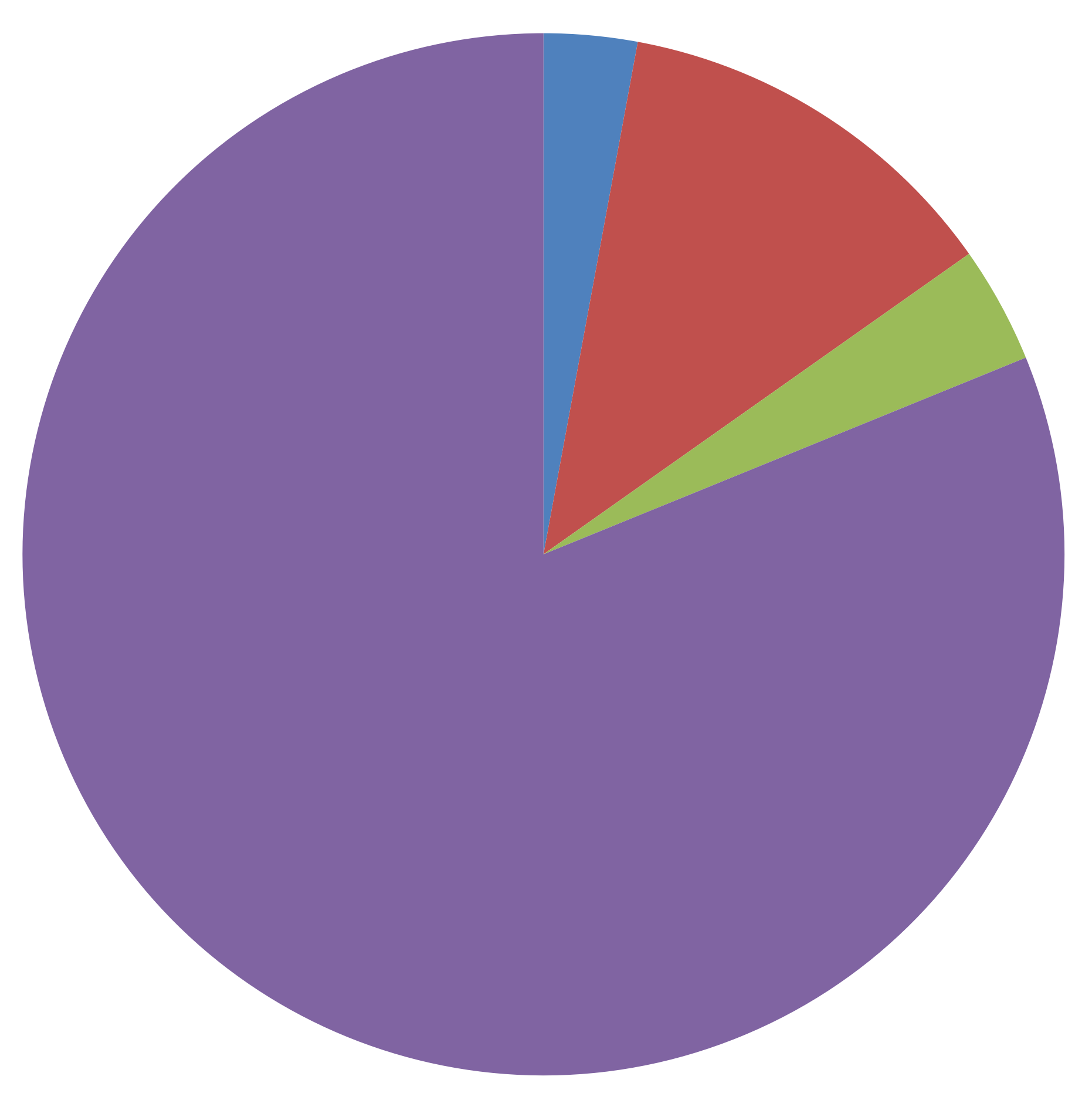

Patients who met DIcriteria in $<8$ hrs

- Patients with DI who did not mee criteria in $<8 \mathrm{hrs}$

- Patients with primary polydipsia who met criteria in $<8 \mathrm{hrs}$

- Patients with primary polydipsia who did not meet criteria in $<8 \mathrm{hrs}$

\section{CONCLUSION}

The standard 8-hour water deprivation test has a limited value in the diagnostic work-up of

$$
\begin{aligned}
& \text { patients with } \\
& \text { polydipsia-polyuria syndrome. }
\end{aligned}
$$

Despite an extended test for up to 48 hours, a partial DI may have been missed in as many as one fourth of the patients diagnosed with primary polydipsia.

For additional information, please contact: Penelope Trimpou E-mail: penelope.trimpou@gu.se 